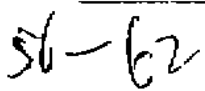

太湖大银鱼生长特性的研究

\author{
王玉芽 蒋全文 $\quad$ S 965.224 \\ \{中国水产科学研究院拨水渔鱼研究中心、无锡 214081)
}

提要本文根据太湖大银鱼 (Protosalanx hvulocrantus Abbott) 周年生物学实测资料,并 应用Von Bertalanffy 生长方程进行计算，对大银鱼在太湖的生长规律及特点进行分析探讨，

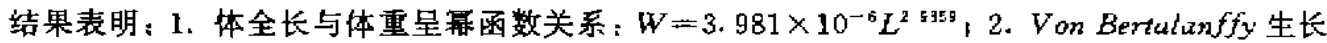
方程的各项参数值为 $1 L_{\infty}=219.86(\mathrm{~mm}), W_{\infty}=28.56(\mathrm{~g}), K=0.1469, t_{0}=0.33$ ； 3. 生

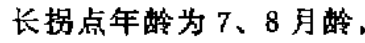

\title{
关镜调 太湖，太银鱼，生长待性
}

太湖大银鱼 (以下简称大银鱼) 是太湖银鱼渔业的主要渔获对象之一，产量占太湖 银鱼渔业年总产量的 $50 \%$ 以上, 产品全部出口创汇, 是太湖的重要经济鱼类。大银鱼的 生物学及种群生态等前人已做了大量研究工作, 但其在太湖的生长规律及特点, 还末见 详细报道。本文通过计算并重点分析水银鱼种群的体全长与体重关系、生长曲线、生长 方程等、旨在阐明大银鱼的生长特性和掌握其生长规律, 为渔业管理提供依据。

\section{一、材料与方法}

1983-1984 年用 $240 \mathrm{Hp}$ 调查船每月定期、定点 (全太湖定八个点)，用航拖锥形银 鱼网采样, 从各点的渔获物中随机取标本 30 尾, 进行体长、体重等生物学测定, 共淄定 1983 世代大银鱼 1200 尾。

大银鱼的寿命为一年, 故在计算、分析中年龄用月龄表示。据调查, 大银鱼在太湖 的熬殖盛期通常在 1 月下旬至 2 月上旬, 眼䏩䛇化期在自然环境中约为一个月左右. 故 本文定 3 月 10 日为零月龄, 4 月 10 日为 1 月龄, 以后类推。相应月龄的体全长与体重数 值见表 1 。

依据上述资料拟合Von Bertalanffy 生长方程, 计算出各项生长参数。

事 1 大锤鱼各月龄的体长与体工

Tab. 1 Body length and weight of the icefish in each month

\begin{tabular}{|c|c|c|c|c|c|c|c|c|}
\hline 日 期 & 4月 10 日 & 5 月 10 日 & 6 月 10 日 & 7月 10 日 & 8月 10 日 & 5 月 10 日 & 10 月 10 日 & 11 月 10 日 \\
\hline 月 峈 & 1 & 2 & 3 & 4 & 5 & 6 & 7 & 8 \\
\hline 全长 (mm) & 21.09 & 47.64 & 72.38 & 91.56 & $109 . \mathrm{E} 1$ & 124.74 & 137.85 & $148.5 U$ \\
\hline 体面 (g) & 0.036 & 0.336 & 1.190 & 2. 349 & 4. 153 & 5.569 & 7.562 & g. 940 \\
\hline
\end{tabular}

.1; 本文承菜仁逵主任、袁蔽文副研究员审阅、谨致谢意。 


\section{（一）体长与体重相关}

\section{二、结 果}

鱼类种群的生长是决定种群数量变动的主要因子, 而生长的一般规律, 主要是指体 长、体重随时间而变化的规律。本文根据大银鱼生物学测定资料, 经点图分析, 其体长 与体重相关如图 1。由图 1 可见, 大银鱼种群体长与体重关系呈量函数关系, 符合 $W=a L^{b}$ 之规律，计算结果体长、体重关系式为：

$$
W=3.981 \times 10^{-\theta} L^{z .9359}, \quad R=0.999
$$

式中 $W$ 为鱼体全重, $L$ 为鱼体全长, $a, b$ 为参数, $R$ 为体长与体重的相关系数。

上述结果表明, 大银鱼种群的体 长与体重呈量函数关系, 且指数 $b$ 近 似于 3 , 即体重与体长的三次方成正 比。可认为种群是匀速生长类型。

\section{（二）鱼体增长氞和各项计萛指标}

相对增长率与生长指标能客观地 反映鱼类在不同生长阶段的生长特 点, 而绝对增长量则可反映以种系发 育的形式定型下来的鱼类个体生长特 点。表 2 为大银鱼的各项生长指标、相 对增长率和绝对增长量。

由表 2 可知, 大银鱼的相对增长 率和体长绝对增长量 1 月龄时最高、 以后随月龄的增加而逐渐下降。尤其 是前四个月问, 各项增长指标参量较 高, 说明大银鱼幼鱼阶段生长发育极 为迅速。但体重的月增长量 1 月龄时 很低, 以后随月龄的增加而增加, 7 月 跉后则又明显下降。

\section{（三）生长方程和生长曲线}

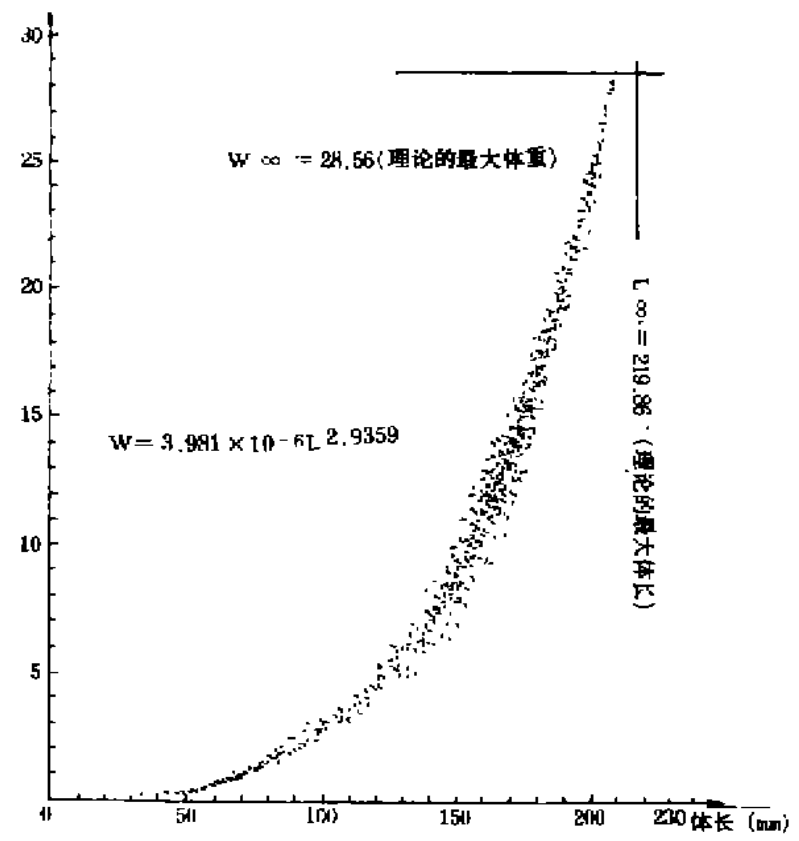

图 1 大银鱼体长与体重的关系

Fig. 1 Relationship body length and weight of icefish

根据表 1 中各月龄的平均体全长 和平均体重的实测值，应用 Von Bertalanffy 生长方程描绘大银鱼的生长曲线。

Von Bertalanffy 生长方程为:

$$
\begin{aligned}
L_{t} & =L_{\infty}\left[1-\mathrm{e}^{-k\left|t-t_{\mathrm{p}}\right\rangle}\right] \\
W_{\iota} & =W_{\infty}\left[1-\mathrm{e}^{-k\left\langle t-t_{\mathrm{q}}\right)}\right]^{3(1)}
\end{aligned}
$$

式中 $L_{t}$ 为七月龄时体长 $(\mathrm{mm}), W_{\mathrm{s}}$ 为七月龄时体重 $(\mathrm{g}), L_{\infty}$ 为渐近体长 $(\mathrm{mm})$, $W_{\infty}$ 为渐近体重 $(\mathrm{g}), t$ 为月龄, $t_{0}$ 为体长和体重为零时的理论年龄, $k$ 为生长曲线的平均 曲率。

(1)大银鱼的生长指数 b 值为 2.9359 , 近似于 3 , 为葺便起见, 体重生长公式的指数 b 也取 3 , 规为等比生长。 
将 (1) 式变换为: $L_{r-1}=L_{m}\left(1-\mathrm{e}^{-K}\right)+\mathrm{e}^{-K} L_{2}$

将 (2) 式变换为: $W_{2+1}^{1 / 3}=W_{r}^{1 / 3}\left(1-\mathrm{e}^{-\boldsymbol{x}}\right)+\mathrm{e}^{-\boldsymbol{x}} W_{\mathrm{r}}^{1 / 3}$

式（3）、（4）即为著名的W alford 法（定差法），如图 2。

变 2 大银鱼的各项生长指标及绝对增长音、相对增长率

Tab. 2 Absolute increment, relative increment and growth indexes of the icefish

\begin{tabular}{|c|c|c|c|c|c|c|c|}
\hline 月 龄 & $\begin{array}{c}\text { 体长范围 } \\
\text { (mm) }\end{array}$ & $\begin{array}{c}\text { 平均体长 } \\
\text { (mm) }\end{array}$ & $\begin{array}{c}\text { 平均性重 } \\
\text { (g) }\end{array}$ & 体长增长量 & $\begin{array}{l}\Delta L / L \\
(\%)\end{array}$ & $\begin{array}{c}\text { 体重㙞长量 } \\
\text { (g) }\end{array}$ & $\begin{array}{c}\Delta w / w \\
(\%)\end{array}$ \\
\hline 1 & $16-27$ & 21 & 0.04 & 27 & 125.80 & 0.80 & 833.0 \\
\hline 2 & $25--51$ & 48 & 0.34 & 25 & 51.53 & 0.85 & 254.0 \\
\hline 3 & $6:-105$ & 72 & 1.19 & 20 & 27.05 & 1.16 & 97.39 \\
\hline 4 & $75-116$ & 92 & 2.35 & 18 & 19.19 & 1.80 & 76.80 \\
\hline$\frac{5}{6}$ & $\frac{87-137}{96-145}$ & $\frac{110}{125}$ & $\frac{4.15}{5.57}$ & 15 & 13.80 & 1. 42 & 34.10 \\
\hline 7 & $193-183$ & 138 & 3.56 & 13 & 10.51 & 1.99 & 35.79 \\
\hline $\mathrm{g}$ & $109-197$ & 149 & 8. 94 & 11 & 7.75 & 1.38 & 18.22 \\
\hline
\end{tabular}

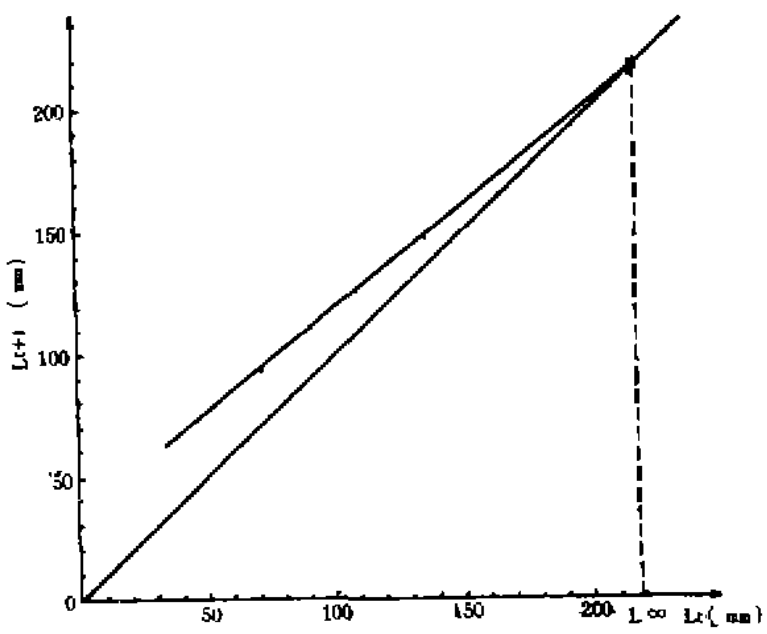

图 2 大银鱼定差图

Fig. 2 Walford curve of the icefish

用表 1 数据进行直线回归, 求出大银鱼的各项生长参数值:

$$
\begin{aligned}
L_{\mathrm{co}}=220(\mathrm{~mm}), \quad K=0.14685, & t_{0}=0.31, \\
W_{\infty}=28.56(\mathrm{~g}), \quad K=0.1566, & t_{0}=0.30
\end{aligned}
$$

由上可见，用体长和体重分别求得的参数值尽管有些差异，但很接近。一般情况下 均取用体长资料求得参数值，然后通过体长与体重的关系式求取 $W$ 。值。

现将实测值与Von Bertalanffy 生长方程的计算值进行比较（表 3)。

由表 3 可见，实测值与计算值基本相符，说明 Von Bertalanffy 生长方程所描述的大 


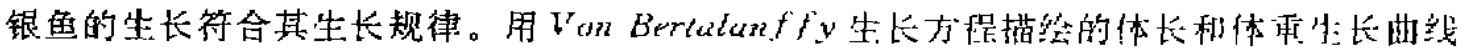

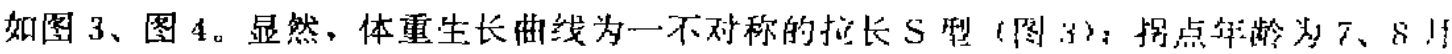

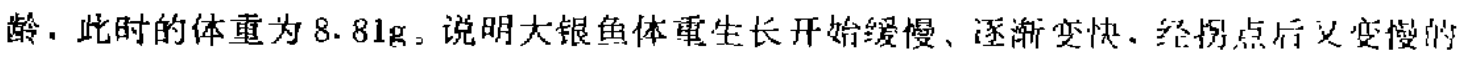

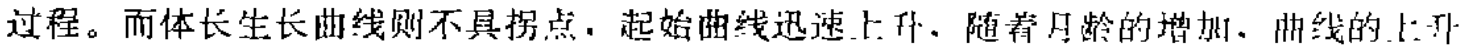
逐渐变缓、趋向渐近值 $L$.. (图 4)。

表方大银鱼体长、体严计篻值与实测值比较

Tab. 3 Comparison of calculated values with really mensured values in body length and body weight of the icefish

\begin{tabular}{|c|c|c|c|c|c|c|c|c|}
\hline $\mathrm{B}$ & 此 & 1 & 2 & $\therefore$ & 1 & 5 & $\vdots$ & 7 \\
\hline 平均体长 & 实测值 & 21 & $\sqrt{4 x}$ & 72 & $! 2$ & 111 & $12 \pi$ & 134 \\
\hline$(\mathrm{mm})$ & 计施 & 21 & 49 & 72 & 92 & 3161 & 125 & $13 k$ \\
\hline 平均体重 & 实测低 & $(1.04$ & 6.31 & 1.14 & 2. 35 & 4.15 & 5.78 & $3.5, A$ \\
\hline$\langle g\rangle$ & 计往 & 0.113 & 0.31 & 1. III & 2.23 & 3.79 & Iו ון & $7 . \therefore$ \\
\hline
\end{tabular}

（四）生长速度和生长加速 庵

研究鱼类的生长速度.可避 免在其讯速生长阶段进行捕获。 从而合理、有效地利用资源。求 得高产。由 $V o n$ Bertulan $f f y$ 生 长方程一次求导, 即得生长速度 方程:

$\mathrm{d} L / \mathrm{d} t=K \cdot L_{1 .} \cdot \mathrm{e}^{-K t / t_{\mathrm{n}} 3}$

$\mathrm{d} W / \mathrm{d} t=b \cdot W_{2} \cdot K \cdot \mathrm{c}^{-K^{\prime} t_{t}-t_{11}^{\prime}}$

$$
\cdot\left[1-e^{-K(t-1) ! 1} \cdot\right]^{b-1}
$$

式中 $\mathrm{d} L / \mathrm{d} t$ 为体长生长 速度, $\mathrm{d} W / \mathrm{d} t$ 为体重生长速度， 再将 (5)，(6) 式求导，即得生 长加速度方程:

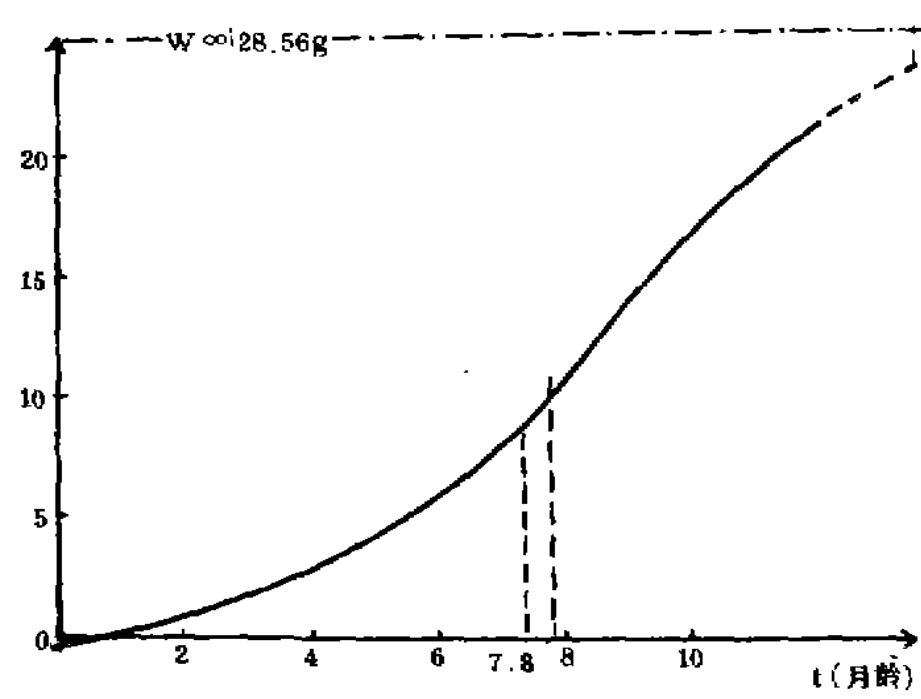

图 3 大银鱼体雷生长曲线

Fig. 3 Growth curve of body weight

$$
\begin{aligned}
& \mathrm{d}^{2} L / \mathrm{d} t^{2}=-L_{\mathrm{n}} \cdot K^{2} \cdot \mathrm{e}^{-K\left(\mathrm{r}-t_{i}\right)}
\end{aligned}
$$

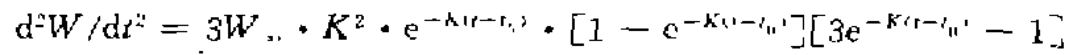

式中 $\mathrm{d}^{2} L / \mathrm{d} t^{2}$ 为体长生长加速度, $\mathrm{d}^{2} W / \mathrm{d} t^{2}$ 为体重生长加速度。

令 (8) 式的 $\mathrm{d}^{2} W / \mathrm{d} t^{i}=0$

$$
\text { 则 } t_{\text {则 }}=t_{0}+\left(L_{\mathrm{n}} b\right) / K
$$

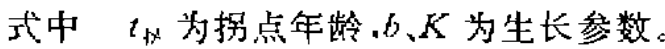

将各生长参数代入式 $(9)$, 求得拐点年蛒为 $7 、 8$ 月龄。

图了、图6为大银鱼的体重生长速度和加速度曲线。

由图5、图6可见, 大银鱼在 $2.2<\mathrm{t}<7.8$ 月龄时, 体重生长速度曲线上升而体重生长 加速度曲线下降. 但 $\mathrm{d}^{2} W / \mathrm{d} t^{2}$ 均为正值, 表明在 $2.2-7.8$ 月龄间. 体重生长速度为遭增 


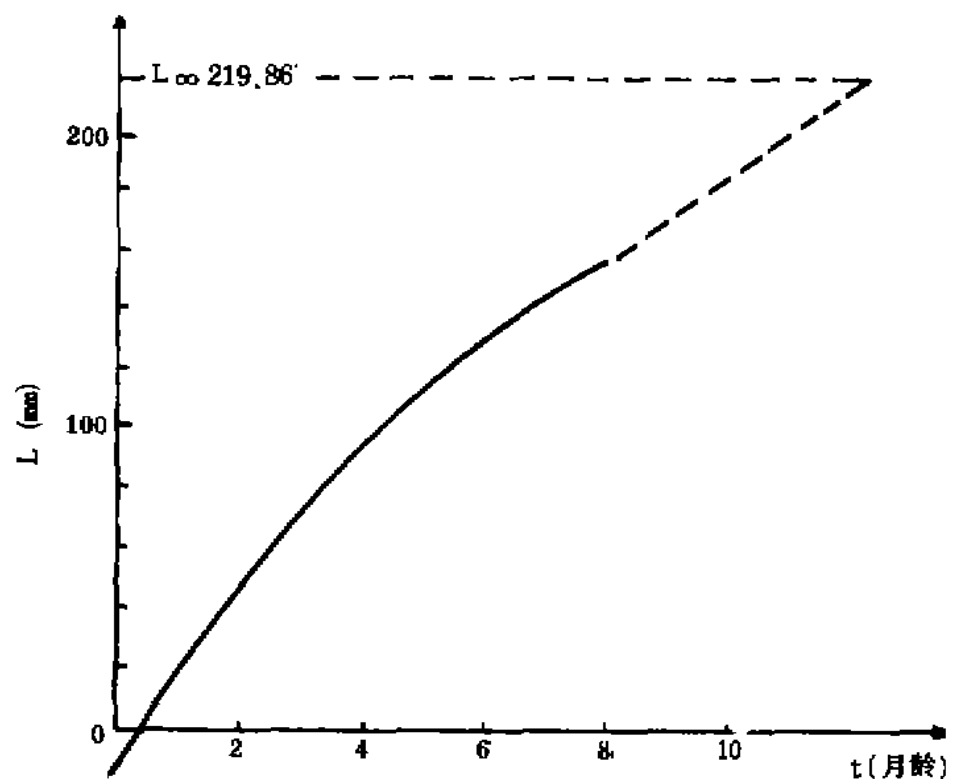

图4 大银鱼生长曲线

Fig. 4 Growth curve of body length

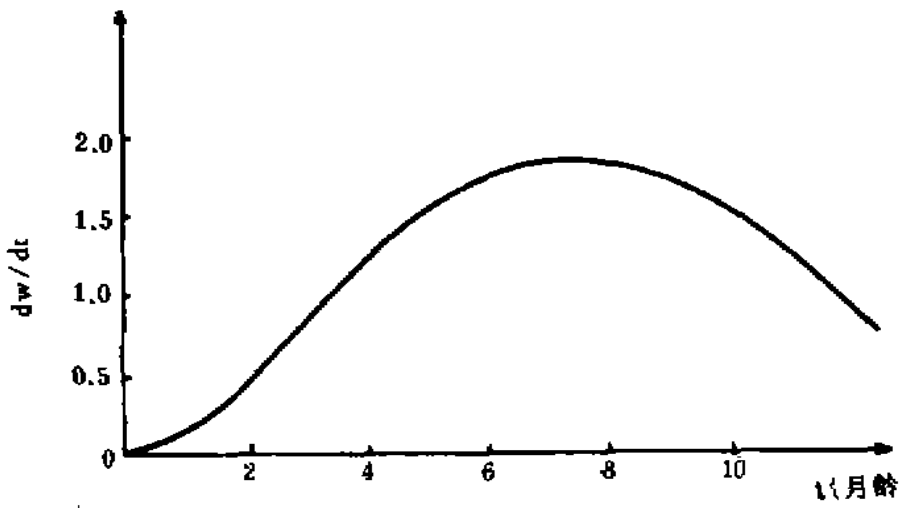

图5 大银鱼体重生长速度曲线

Fig. 5 Growth rate curve of body weight

阶段，递增的速度逐渐下降; 但 当 $\mathrm{t}>7.8$ 月龄时, 体重生长速度 曲线和体重生长加速度曲线均 趋下降, 且 $\mathrm{d}^{2} W / \mathrm{d} t^{2}$ 为负值, 表 明自7.8月龄之后, 体重生长速 度为递㺂阶段, 加速度从椡负 值, 随着月龄的增加, 最后又趋 于 0 。由图6还可见，加速度为负 苴区内, 月龄增加, 曲线有回升 的趋势, 可认为长到极限时, 自 然加速度为0。

由图7、图8可见，体长生长 速度随着月龄的增加而下降, 最 后渐趋于0；而体长生长加速度 诒终为负值, 随着月龄的增加而 增加, 说明体长生长速度随着月 龄增加渐趋缓慢。

\section{三、小 结}

1. 大银鱼体全长与体重相 关关系为幂函数关系，关系式 为:

$$
W=3.981 \times 10^{-6} L^{2.9359},
$$
$R=0.999$ 。

2. 大银鱼幼鱼阶段生长发 育极为迅速，元其是前四个月龄。 各项生长指标参量均较离；8月 龄后体长、体重生长速度逐斩变 慢。

3. 大银鱼的生长拐点年龄为7.8月龄, 即10月份。据调查, 10月份大银鱼性腺发育为 I 期, 故可认为其拐点年龄是性腺开始发育的一个临界点, 也是其生长由迅速变缓慢的 一个转折点。 


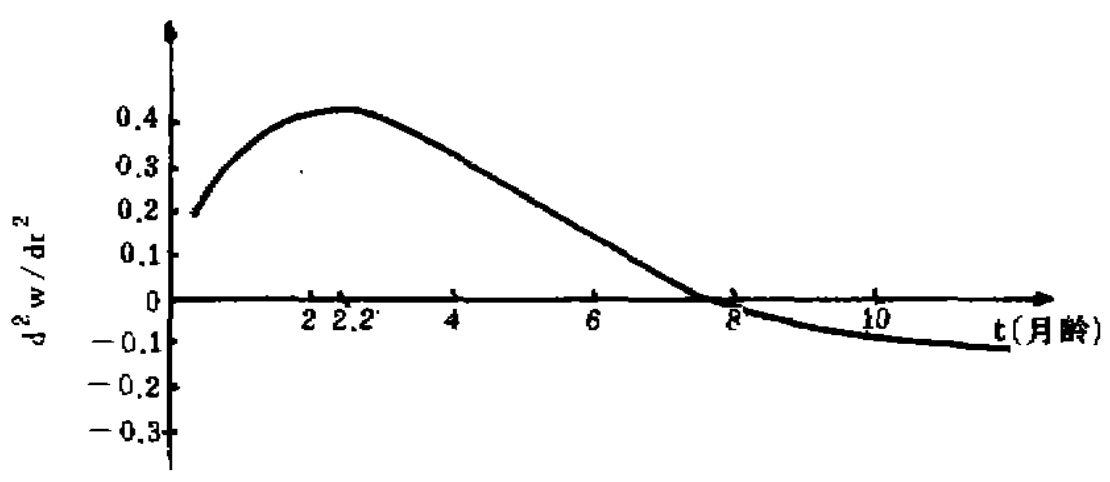

图6 大银鱼体重生长加速度曲线

Fig. 6 Accelerated growth curve of body weight

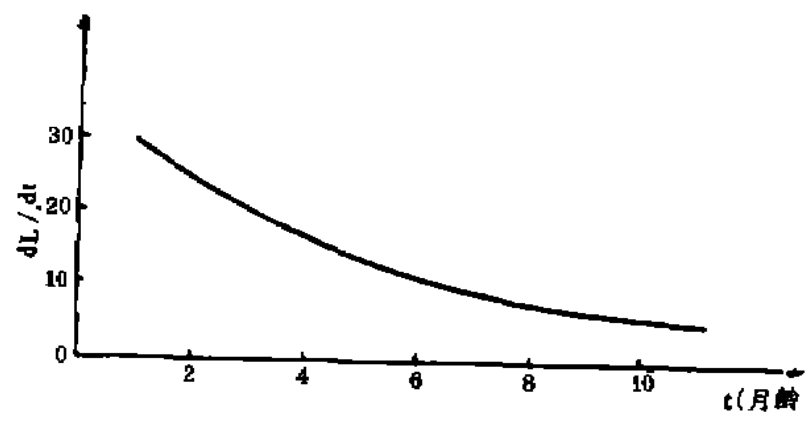

图7 大银鱼㤓长生长速度曲线

Fig. 7 Growth rate curve of body length

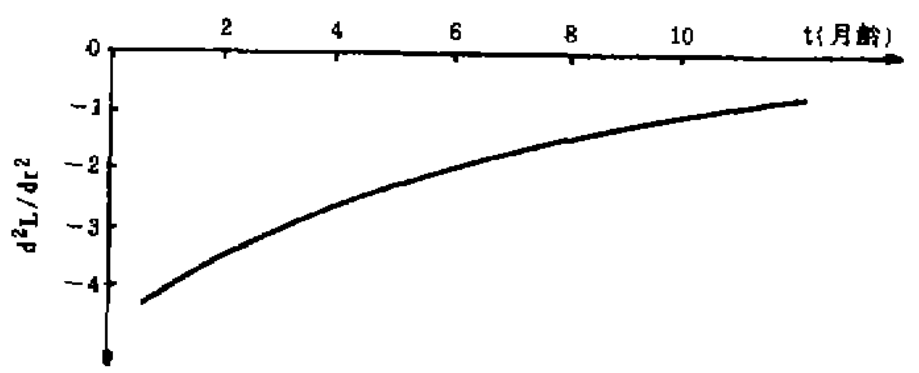

图8 大银鱼体长生长加速度曲线

Fig. 8 Accelerated growth curve of body length 


\title{
参 考 献
}

[1] 陈宁生。太湖所产银鱼的初步研究。水生生物学峲刊, (2)，1956、324一334。

[2」张开等。法湖产大银鱼生物学改其增殖的研究。水产学振，1981，5(1)：29-39.

[3] 李星颉等,鱼类生长的数学描述。浙江水产学院学提，1983，2(1): 29-39.

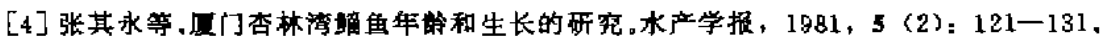

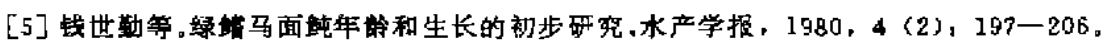

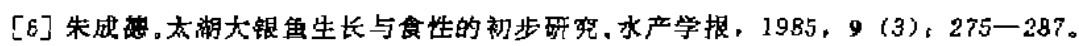

\section{RESEARCH OF GROWTH PROPERTIES OF PROTOSALANX HYALOCRANIUS ABBOTT}

\author{
Wang Yufen Jiang Quanwen \\ (Freshwater Fishertes Research Centre of the Chenese Acadenty of Fisheries Sciences, Wuxi 214081 )
}

\begin{abstract}
According to the biological data of Protosalanx kyalocranius Abbott measured annully, this paper presents its growth properties, growth curves which are fit with Von Bertalanffy growth model and resources properties of the population. The following conclusion has been drawn;

1. Body length and body weight have relation to power function

$$
W=3.981 \cdot 10^{-6} \cdot L^{2.8395}, \quad R=0.999 \text { 。 }
$$

2. The parameters of Von Bertalanffy growth formula are:

$$
L_{\infty}=219.86(\mathrm{~mm}), \quad K=0.1469, t_{0}=0.33, W_{\infty}=28.56(\mathrm{~g}) 。
$$

3. Age of growth inflection point is 7.64 month-age, while body weight equals to one-third of asymptotic weight.

4. Considering growth properties of the icefish only, it is better that fishing season begins in the month of inflection point of month-age.
\end{abstract}

key words Taihu lake, Protosalanx hyalocranius, growth 